\title{
La présence à distance en e-Formation Entretien avec Annie Jézégou
}

\author{
Presence at a distance in e-Learning: \\ Interview with Annie Jézégou
}

\section{Presencia a distancia en e-Learning: Entrevista con Annie Jézégou}

Sonia Androwkha, doctorante en Sciences de l'Éducation et de la Formation Laboratoire CIREL (EA 4354). Université de Lille, France

sonia.proust@univ-lille.fr

RÉSUMÉ

Annie Jézégou est Professeure des Universités en Sciences de l'Education et de la Formation à l'Université de Lille (France), au laboratoire CIREL (Centre Interuniversitaire de Recherche en Education de Lille) et au département SEFA (Sciences de l'Education et Formation des Adultes). L'objet général de ses travaux porte sur les dimensions technico-pédagogiques et socio-pédagogiques qui permettent de favoriser l'autodirection des apprenants en eFormation. Ses recherches sur la e-Formation ont notamment permis d'étayer au plan théorique le concept d'ouverture des environnements numériques d'apprentissage. Elles ont également contribué à modéliser le phénomène de la présence à distance en contexte d'eFormation.

Mots-clés : présence à distance, affordance, autodirection

ABSTRACT

Annie Jézégou is Professor of Education and Training Sciences at the University of Lille (France) at the CIREL laboratory (Centre Interuniversitaire de Recherche en Education de Lille) and at the SEFA department (Sciences of Adult Education and Training). The general aim of her work is to address the technical-pedagogical and socio-pedagogical dimensions that promote the self-direction of learners in e-learning. Her research on e-Learning has, in 
particular, made it possible to provide theoretical support for the concept of opening up digital learning environments. It has also contributed to modelling the phenomenon of distance presence in an e-learning context.

Keywords: telepresence, affordance, self-direction

RESUMEN

Annie Jézégou es profesora de Ciencias de la Educación y de la Formación en la Universidad de Lille (Francia), en el laboratorio CIREL (Centro Interuniversitario de Investigación en Educación de Lille) y en el departamento SEFA (Ciencias de la Educación y la Formación de Adultos). El objetivo general de su trabajo es abordar las dimensiones técnico-pedagógicas y socio-pedagógicas que promueven la auto-orientación de los alumnos en el aprendizaje digital. Sus investigaciones sobre el aprendizaje digital han permitido, sobre todo, apoyar teóricamente el concepto de apertura de los entornos digitales de aprendizaje. También ha contribuido a modelar el fenómeno de la presencia a distancia en un contexto de aprendizaje digital.

Palabras clave: telepresencia, affordance, auto-orientación

Annie Jézégou est Professeure des Universités en Sciences de l'Education et de la Formation à I'Université de Lille (France), au laboratoire CIREL (Centre Interuniversitaire de Recherche en Education de Lille) et au département SEFA (Sciences de l'Education et Formation des Adultes).

L'objet général de ses travaux porte sur les dimensions technico-pédagogiques et socio-pédagogiques qui permettent de favoriser l'autodirection des apprenants en e-Formation. L'expression " e-Formation » renvoie à

« des environnements d'apprentissage en ligne dont une des principales propriétés est d'utiliser des technologies du multimédia et l'Internet pour faciliter l'accès à des ressources et des services éducatifs. Ces environnements intègrent des outils logiciels qui permettent la gestion et le suivi d'une formation en ligne, l'accès à des ressources pédagogiques médiatisées, des possibilités technologiques d'interactions synchrones et asynchrones, de travail et de collaboration à distance ou encore de production et de partage de contenus ». (Jézégou, 2019a, p.9).

Ses recherches sur la e-Formation ont notamment permis d'étayer au plan théorique le concept d'ouverture des environnements numériques d'apprentissage. L'ouverture est caractérisée par le degré de liberté de choix accordé à l'apprenant pour qu'il puisse exercer son autodirection, à la fois aux plans motivationnel et métacognitif (autorégulation). Elles ont également contribué à modéliser le phénomène de la présence à distance en contexte d'e-Formation.

Pour la revue Médiations et Médiatisations, Annie Jézégou a accepté de croiser plusieurs de ses apports scientifiques sur la présence à distance en e-Formation avec la thématique de ce numéro, intitulé "Téléprésence, visioconférence ou webconférence: enseignement et apprentissage synchrone et distant ». Nous la remercions pour l'entretien qu'elle nous a accordé. 


\section{SONIA ANDROWKHA : Le titre de ce numéro est "Téléprésence, visioconférence ou webconférence : enseignement et apprentissage synchrone et distant ". Que vous évoque cet intitulé au regard de vos propres travaux de recherche?}

ANNIE JÉZÉGOU : II m'évoque en particulier la question de la présence à distance en lien avec l'usage des Technologies Numériques de l'Information et de la Communication (TNIC) en contexte d'e-Formation (e-learning, blended-learning, MOOC, apprentissage mobile, etc.). Dans mes recherches, cette présence à distance est définie comme la résultante d'une dynamique relationnelle médiatisée entre les apprenants, entre les apprenants et l'enseignant (ou le formateur) au sein d'un espace numérique de communication (Jézégou, 2019b). C'est selon moi la manière la plus large et la plus simple de la caractériser.

Voici, de façon très synthétique, les quelques croisements que je peux opérer entre l'intitulé de ce numéro et mes recherches sur la présence à distance en e-Formation.

Ces dernières abordent principalement la téléprésence, la visioconférence ou la webconférence comme des artéfacts de communication synchrone qui doivent être activés socialement par les apprenants et les enseignants (et formateurs) pour devenir des " espaces numériques de communication ». Sans cette activation, ces artéfacts conservent le simple statut d'outils et de services mis à disposition. Cela vaut également pour tout autre artéfact de communication, comme par exemple les forums, les messageries électroniques, les discussions synchrones en ligne ou encore les réseaux sociaux.

II me semble important d'insister notamment sur deux aspects de ces artéfacts de communication (synchrone ou asynchrone). D'une part, leur activation sociale dépend de leurs propres affordances socionumériques; en effet, ces affordances induisent l'usage (ou le non-usage) qui en est fait. D'autre part, cet usage dépend aussi de l'agentivité, à la fois individuelle et collective, des utilisateurs (en l'occurrence des apprenants et de l'enseignant ou du formateur), notamment à des fins d'activation sociale.

Pour qu'une présence à distance puisse se créer au sein d'un espace numérique de communication, ces deux aspects doivent être réunis. Il s'agit d'une condition nécessaire mais néanmoins insuffisante, cela pour une raison assez évidente : la présence résulte d'une dynamique relationnelle et médiatisée. Par conséquent, elle implique que les apprenants (et l'enseignant) soient suffisamment motivés pour interagir entre eux et qu'ils développent des stratégies efficaces pour qu'une telle dynamique émerge et se développe. Là également, ils doivent faire preuve d'agentivité.

\section{SONIA ANDROWKHA : Pouvez-vous préciser davantage ce que recouvre l'affordance socio- numérique d'un artéfact de communication?}

ANNIE JÉZÉGOU : Avant toute chose, il est utile de préciser ce qu'est un artéfact. Au sens large, un artéfact est un objet matériel fabriqué par l'être humain. Par conséquent, la téléconférence, la visioconférence ou encore la webconférence sont des artéfacts. Ils peuvent être qualifiés de socio-numériques, car il s'agit d'outils et de services numériques dédiés à la communication médiatisée. En toute logique, ces artéfacts socio-numériques entrent dans la catégorie des artéfacts technologiques. Quant au terme d'affordance, il est issu du verbe anglais « to afford » qui signifie à la fois « fournir, procurer » et « être en mesure d'agir »; si cette traduction n'apporte pas vraiment d'éléments tangibles sur le sens original donné à l'affordance, elle permet à tout le moins d'identifier son étymologie en tant qu'anglicisme. Pour aller à 
l'essentiel, je dirais que l'affordance renvoie aux propriétés des artéfacts telles que pensées et élaborées par leurs concepteurs. Ces propriétés peuvent porter sur le design, l'interface, l'ergonomie, la navigation, l'interactivité, etc. En même temps, l'affordance intègre aussi la perception que les utilisateurs ont de l'utilité de ces propriétés pour réaliser une activité donnée ainsi que la perception qu'ils ont de la facilité d'utilisation des artéfacts. En résumé, les artéfacts socio-numériques de communication que sont par exemple la téléconférence, la visio-conférence et la webconférence possèdent certaines affordances socio-numériques. Elles dépendent à la fois de leurs propriétés et de ces deux perceptions singulières propres à chaque l'utilisateur.

Mais le plus important peut-être à retenir est que ces perceptions (d'utilité et de facilité d'utilisation) induisent l'attitude de l'utilisateur à l'égard de l'artéfact qui, à son tour, influe sur son intention de l'utiliser ou pas. Elles agissent également sur la manière dont il utilise l'artéfact en question. Tous ces aspects de l'affordance, que je viens de préciser de façon très synthétique, ont été mis en relief par plusieurs chercheurs dont, pour les principaux, Davis (1989), Gaver (1991) et Norman (1999).

\section{SONIA ANDROWKHA : Quelles sont alors les affordances socio-numériques propices à créer une présence à distance?}

ANNIE JÉZÉGOU : La situation d'affordance dite « perçue » est celle qui contribue à favoriser l'émergence d'une présence à distance en e-Formation. Pour énoncer cela, je m'appuie sur les travaux de Gaver (1991) et ceux de Norman (1999). Ce dernier a montré que les propriétés d'un artéfact technologique suggèrent aussi des possibilités d'utilisation. La question est alors de savoir si un utilisateur potentiel perçoit ou non ces possibilités. Concernant cette troisième perception (en sus de celles d'utilité et de facilité d'utilisation), Gaver (1991) a révélé quatre situations qui peuvent s'appliquer aux artéfacts de communication dont la téléprésence, la visio-conférence ou encore la webconférence. Deux d'entre-elles ne sont pas affordantes : la "fausse » affordance correspond à une situation où l'utilisateur perçoit une possibilité d'utilisation de l'artéfact pour communiquer avec les autres, bien que cette possibilité n'ait pas été prévue par les concepteurs. La situation de "rejet correct » se caractérise, elle, par le fait que l'utilisateur ne perçoit pas les possibilités d'utilisation de l'artéfact pour communiquer, car elles n'ont pas été prévues par les concepteurs. Les deux autres situations sont dites "affordantes »: la première est celle où les possibilités d'utilisation de l'artéfact à des fins de communication ne sont pas perçues par l'utilisateur bien qu'elles aient été proposées par les concepteurs; il s'agit alors d'une affordance "cachée ». Dans la seconde, il perçoit les possibilités d'utilisation de l'artéfact, telles que préalablement mises à disposition par les concepteurs; non seulement il les perçoit, mais il les active pour communiquer avec les autres. Cette situation est qualifiée d'affordance « perçue ».

C'est en particulier ce type de situations qui est favorable à l'émergence et au développement d'une présence à distance en e-Formation, pour une raison somme toute assez logique : la présence à distance résulte d'une dynamique relationnelle médiatisée entre les apprenants, entre ces derniers et l'enseignant (Jézégou, 2019b). Elle nécessite une condition évidente : qu'ils utilisent les artéfacts socio-numériques de communication mis à leur disposition (téléprésence, visioconférence, webconférence ou autres), ce qui interpelle à nouveau la question des affordances socio-numériques de ces artéfacts. En résumé, les apprenants et l'enseignant (ou le formateur) doivent respectivement percevoir et activer les possibilités de communication à distance de ces artéfacts, les juger utiles et faciles d'utilisation. Par conséquent, la situation la plus favorable est bien celle d'une affordance " perçue » telle que décrite précédemment. 


\section{SONIA ANDROWKHA : Outre le rôle joué par cette affordance perçue, quel est celui exercé par l'agentivité des apprenants?}

ANNIE JÉZÉGOU: La situation d'affordance socio-numérique "perçue » est certes une condition nécessaire pour un usage effectif des artéfacts en tant qu'espaces numériques de communication. Mais, comme je l'ai souligné au début de cet entretien, une autre condition, non des moindres, entre également en jeu : celle liée à l'agentivité individuelle et collective des interlocuteurs (apprenants et enseignant/formateur) à développer une dynamique relationnelle médiatisée et porteuse de présence à distance (Jézégou, 2019c). Au plan individuel, l'agentivité s'exprime à la fois par l'intentionnalité d'une personne à agir et le contrôle conscient qu'elle exerce sur la conduite de l'action (Bandura, 2006). L'agentivité interpelle en particulier ses capacités à l'autodirection, c'est-à-dire ses capacités à diriger ellemême l'action en question. Plus précisément, l'autodirection nécessite d'une part d'être motivé à l'égard de cette action, d'autre part d'en contrôler le déroulement par la mise en œuvre de stratégies efficaces d'autorégulation (Jézégou, 2014a). Tous ces aspects liés à l'agentivité individuelle s'appliquent aussi à l'agentivité collective (Bandura, 2006; Jézégou, 2019c).

Si l'action consiste à mettre en œuvre une dynamique relationnelle (qu'elle soit ou non médiatisée), en proximité physique ou en distance géographique), alors les apprenants doivent s'engager individuellement et collectivement dans cette action spécifique et y persévérer dans le temps, cela de façon intentionnelle. Ils doivent aussi développer des stratégies à la fois individuelles et collectives pour développer cette dynamique. De même, l'enseignant doit faire preuve d'agentivité pour soutenir les efforts des apprenants dans ce sens. Sans cette agentivité, aucune présence à distance ne peut se créer au sein d'un espace numérique de communication (Jézégou, 2019c).

\section{SONIA ANDROWKHA : Si ces deux conditions d'affordance des artéfacts socio-numériques et d'agentivité des apprenants et de l'enseignant sont réunies, comment se manifeste alors "la présence à distance "?}

ANNIE JÉZÉGOU : Comme déjà précisé à plusieurs reprises, la présence à distance résulte d'une dynamique relationnelle médiatisée entre les apprenants, entre les apprenants et l'enseignant (le formateur). Cette définition, somme toute assez générale, rejoint celle formulée par d'autres chercheurs dont, pour les principaux, Garrison et ses collaborateurs (Garrison et Anderson, 2003; Garrison et Archer, 2007; Garrison, 2016) et Whiteside $(2015,2017)$. Néanmoins, la comparaison avec mes propres travaux s'arrête là. En effet, je n'aborde pas la présence de la même manière. D'une part, je lui attribue des ancrages épistémologiques et théoriques différents; d'autre part, je ne mobilise pas le même réseau de concepts. Par conséquent, le modèle théorique que je propose (Jézégou, 2012, 2013, 2014b, 2019b) diffère significativement de ceux proposés par ces collègues anglophones nord-américains.

Dans mes recherches, la présence à distance se réfère à une situation particulière au regard d'un but commun : le groupe d'apprenants conçoit et met en œuvre collectivement des activités visant à résoudre une situation problématique (Jézégou, 2012, 2014b, 2019b). J'emprunte à Dewey (1938) cette expression générique de "situation problématique » pour signifier un travail de groupe dont la finalité est par exemple de mener un projet, répondre à un évènement inattendu, trouver une solution à un problème, etc. Lors de la démarche collective de ce travail en groupe, certaines formes d'interactions sociales entre les apprenants, entre ces derniers et l'enseignant, permettent de créer une présence sociale à distance, cela malgré leur éloignement géographique. Depuis plusieurs années, mes recherches visent à caractériser ces interactions, à spécifier la manière dont elles se manifestent au sein d'un espace numérique de communication et à identifier le rôle joué par les affordances socio-numériques des artéfacts ou encore 
celui de l'agentivité individuelle et collective. Les résultats de ces travaux vont paraître en 2020 dans mon prochain ouvrage sur « la présence à distance en e-Formation ».

D'ores et déjà, un premier jalon important de ce programme de recherches a été publié (Jézégou, 2012, 2013, 2014b, 2019b). Ces publications présentent les fondements épistémo-théoriques du méta-concept de présence et en propose une modélisation théorique dans laquelle la présence est déclinée en trois dimensions constitutives : (1) la présence socio-cognitive, (2) la présence socio-affective, (3) la présence pédagogique.

\section{SONIA ANDROWKHA : Comment caractérisez-vous ces trois formes de présence?}

ANNIE JÉZÉGOU : La caractérisation de ces trois formes de présence est obtenue à partir d'un travail d'identification des processus interactionnels à l'œuvre dans chacune d'entre-elles. Je les définis respectivement de la manière suivante en contexte d'e-Formation (Jézégou, 2012) :

La présence socio-cognitive résulte des transactions médiatisées entre les apprenants, c'est-à-dire des interactions sociales de confrontation de leurs points de vue, d'ajustements mutuels, de négociations et de délibérations pour résoudre de façon commune et conjointe une situation problématique.

La présence socio-affective résulte des interactions sociales médiatisées qui permettent de créer un climat socio-affectif favorable aux transactions entre les apprenants, notamment celles liées à la cohésion, à la symétrie de la relation et à l'aménité.

La présence pédagogique résulte des interactions sociales médiatisées que le formateur entretient avec les apprenants pour soutenir les transactions entre les apprenants tout en contribuant à un climat socioaffectif favorable. Ces interactions s'expriment lors d'activités de coordination, d'animation et de modération auprès des apprenants lors de la démarche de résolution commune et conjointe d'une situation problématique.

Les fondements épistémo-théoriques qui ancrent chacune de ces trois définitions sont développés depuis 2012 dans plusieurs de mes publications. Il serait vraiment trop long de les présenter à nouveau dans cet entretien. A minima, je dirais ici que ces fondements se réfèrent à la fois à la perspective transactionnelle de la philosophie du pragmatisme développée par Dewey et Bentley (1949), à la théorie du conflit sociocognitif issue de l'approche européenne du socio-constructivisme (Darnon, Butera et Mugny, 2008; Monteil, 1987; Perret-Clermont et Nicolet, 2002), à la conception dite contradictoire de la collaboration (Damon et Phelps, 1989) ou encore aux travaux en psychologie sociale sur les dynamiques de groupe et la communication humaine (Amado et Guittet, 2017; Albric, 2019; Lewin, 1947). Le modèle de la présence à distance que je propose est une construction théorique ancrée dans ces fondements épistémothéoriques. Elle s'est édifiée à partir de la mise en réseaux de concepts phares et systématiquement définis. II s'agit notamment des concepts de distance, de proximité, de tiers-lieu, de relation, d'interaction, de transaction, de groupe, de médiatisation, d'affordance, d'agentivité, de collaboration, de conflit sociocognitif, de cohésion, etc. La liste est relativement longue...

Le modèle émet également plusieurs énoncés étayés et argumentés au plan théorique et conceptuel. L'un d'entre eux soutient que les trois présences, telles de définies précédemment, contribuent ensemble à l'émergence et au développement d'une communauté d'apprentissage en ligne, tout en favorisant la construction individuelle et collective de connaissances. Le modèle est formalisé par une représentation schématique qui permet de saisir rapidement les processus interactionnels à l'œuvre au regard de 
chacune des trois dimensions de la présence ainsi que le fonctionnement général du phénomène (Jézégou 2012, 2014b, 2019a). Mon prochain ouvrage est principalement dédié à l'explicitation de ce modèle.

\section{SONIA ANDROWKHA : Vous insistez souvent sur le fait que la présence à distance, ainsi que les trois formes qu'elle recouvre, est perçue et vécue. Pouvez-vous préciser?}

ANNIE JÉZÉGOU : La présence à distance, vue sous l'angle global ou de celui de chacune de ses trois dimensions, est à la fois perçue et vécue. En effet, les apprenants peuvent respectivement ressentir de façon métaphorique la présence de leurs interlocuteurs (pairs et enseignant/formateur), cela malgré leur séparation physique et grâce aux interactions qu'ils entretiennent via l'usage des artéfacts socionumériques de communication. Ce ressenti leur permet alors d'atténuer la perception de distance a minima géographique qui les sépare des autres pour vivre une sensation de proximité. De même, la qualité et la multiplicité des interactions sociales médiatisées entre les apprenants séparés physiquement peuvent aussi induire un sentiment partagé et collectif de présence, et par là même concrétiser une proximité ressentie (Jézégou, 2019b). Pour exemple, les apprenants peuvent ressentir, penser et apprécier la présence pédagogique du formateur, jusqu'à le sentir proche, en raison de la réactivité dont ce dernier fait preuve dans ses réponses. Par conséquent, ce sentiment de présence "à distance " est une représentation fantasmée de la relation à autrui. Elle génère une proximité consciente dans l'imaginaire, tout en relevant principalement de formes émotionnelles et de ressentis socio-affectifs qui, eux, sont bien réels; la présence subjective est soutenue et animée par une représentation cognitive construite à partir d'un vécu sensoriel dans le rapport à l'autre. A ces aspects liés à la présence subjective d'autrui s'ajoute la perception de sa propre présence à l'autre, c'est-à-dire " d'être là » pour cet autre en tant que personne réelle et « d'être là ensemble » au sein d'un espace numérique de communication.

Le plan objectif de la présence à distance est, quant à lui, différent de la dimension subjective, sans pour autant en être exempt. En effet, un "sentiment de présence » peut cohabiter avec une présence observable, tangible et mise en actes dans les faits. Ces deux facettes de la présence ne s'opposent pas. Elles se complètent, tout en se référant à deux univers différents : d'une part, un univers intérieur empreint de ressentis et d'imaginaire; d'autre part, un univers concret inhérent à l'action. Ainsi, la présence objective se construit grâce à certaines formes d'interactions sociales entre les apprenants, entre ces derniers et l'enseignant (ou le formateur). Ces formes spécifiques d'interactions ont été décrites, dans ce qu'elles ont d'essentiel, dans chacune des trois dimensions de la présence (socio-cognitive, socio-affective, pédagogique). Elles se mettent en œuvre lors des activités à réaliser en groupe pour résoudre de façon collaborative une situation problématique. En sus de l'impression " d'être là » et " d'être là ensemble », la présence résulte du « faire là » et du « faire là ensemble ». Le " faire là » est la condition individuelle d'une présence en actes dans un espace numérique, car il permet à l'apprenant d'y exister. Le «faire là ensemble " se situe, quant à lui, dans le registre d'un collectif réalisant des activités communes et témoigne de l'existence d'une présence dans cet environnement.

En résumé, je dirais que la perception de présence et la présence objective cohabitent. Mais celle à laquelle s'intéressent plus particulièrement mes travaux est la présence objective, vécue et en actes.

\section{SONIA ANDROWKHA : En quoi cette présence est-elle réelle et non virtuelle?}

ANNIE JÉZÉGOU : J'insiste beaucoup sur le fait que la présence à distance en e-Formation est bien réelle en effet, tout comme chacune de ses trois dimensions. Pour aller ici à l'essentiel, je dirais que, depuis l'avènement des Technologies Numériques de l'Information et de la Communication (TNIC), le terme 
« virtuel » est couramment mentionné dans nombre de configurations, telles que par exemple un environnement virtuel d'apprentissage, un espace virtuel de communication ou encore une communauté virtuelle d'apprentissage. Le recours à ce terme est légitimé le plus souvent par le fait que ces configurations existent et se déploient notamment grâce à l'usage d'artéfacts socio-numériques. Certes, un tel usage nécessite une infrastructure matérielle (ordinateurs, réseaux informatiques, logiciels, etc.) ainsi que des contenus et des relations médiatisées, donc immatérielles. Mais cette assimilation du virtuel au numérique est simpliste et contestable. En effet, elle s'appuie le plus souvent sur une opposition entre " virtuel » et « réel », le réel faisant ici référence au monde physique, tangible, au concret ou encore à l'objet palpable, cela a contrario du virtuel, porteur d'immatérialité grâce au numérique. Cette opposition est largement adoptée par le sens commun, parfois même relayée par la recherche en Sciences humaines et sociales. Pour ma part, je n'adhère pas à cette conception parfois qualifiée de " dualiste numérique " (Jurgenson, 2012).

En effet, je défends la position selon laquelle un espace de communication constitué par des artéfacts socio-numériques est un « lieu réel » soumis à des temporalités synchrones et/ou asynchrones. II héberge des activités humaines médiatisées de mise à disposition, de production, d'enrichissement et de mutualisation de ressources numériques ainsi que d'interactions sociales interpersonnelles et groupales. Ces interactions sociales sont contextualisées, évolutives et finalisées à la fois par des intérêts personnels et par un but partagé d'apprentissage. En tant que supports humains de la relation médiatisée, elles sont soumises à l'affordance socio-numérique des artéfacts technologiques qui composent cet espace numérique de communication. Dans tous les cas, la présence se réfère à une dynamique relationnelle entre ces sujets psychologiques et sociaux, grâce à l'exercice de leur agentivité et à leur usage efficace des artéfacts de l'espace numérique de communication. La présence ainsi créée et vécue n'est donc pas virtuelle; elle est bien réelle au même titre que l'espace au sein duquel elle se manifeste.

\section{Liste de références}

Amado, G. et Guittet, A. (2017). Dynamiques des communications dans les groupes. Armand Colin.

Albric, J. C. (2019). Psychologie de la communication : théories et méthodes. Dunod.

Bandura, A. (2006). Toward a Spychology of Human Agency. Perspectives on Psychological Science, 1(2), 164-180.

Dewey, J. (1966). Logique. La théorie de l'enquête (G. Deledalle, trad.). PUF (ouvrage original publié en 1938 sous le titre The Theory of Inquiry chez Southern University Press).

Dewey, J., et Bentley, A. F. (1949). Knowing and the known. Dans A. Boydston, John Dewey: the later works. 1925 - 1953 (vol. 16). Southern Illinois University Press.

Damon, W. et Phelps, E. (1989). Critical distinctions among three approaches to peer education. International Journal of Educational Research, 3(1), 9-19.

Darnon, C., Butera, F. et Mugny, G. (2008). Des conflits pour apprendre. Presses Universitaires de Grenoble.

Davis, F. D. (1989). Perceived Usefulness, Perceived Ease of Use, and User Acceptance of Information Technology. MIS Quarterly, 13 (3), 319-340.

Garrison, R. (2016). Thinking Collaboratively: Learning in a Community of Inquiry. Routledge.

Garrison, D. R. et Anderson, T. (2003). E-learning in the $21^{\text {st }}$ Century: A Framework for Research and Practice. Routledge.

Garrison, D. R. et Archer, W. (2007). A theory of Community of Inquiry. Dans M.G. Moore (dir.), Handbook of Distance Education, 2, 77-88. Lawrence Erlbaum Associates.

Gaver, W. W. (1991). Technology affordances. Dans Proceedings of the SIGCHI Conference on Human Factors in Computing Systems (pp. 79-84). ACM.

Jézégou, A. (2012). La présence en e-learning : modèle théorique et perspectives pour la recherche. International Journal of E-Learning \& Distance Education (IJEDE), 26(1). http://ijede.ca/index.php/jde/article/view/777/1409 
Jézégou, A. (2013). La présence en e-learning : une dimension socio-éducative pour favoriser l'autodirection des apprenants. Dans P. Cyrot, C. Jeunesse, et D. Christol (dir.) Renforcer l'autoformation : aspects sociaux et dimensions pédagogiques (p.145-158). Chronique sociale.

Jézégou, A. (2014a). L'agentivité humaine : un moteur essentiel pour l'élaboration d'un environnement personnel d'apprentissage. Sciences et Technologies de I'Information et de la Communication pour l'Éducation et la Formation, 21. http://sticef.univ-lemans.fr/num/vol2014/17-jezegou-epa/sticef 2014 NS jezegou 17.htm

Jézégou, A. (2014b). Le modèle de la présence en e-learning. Une modélisation théorique au service de la pratique, notamment en contexte universitaire. Dans G. Lameul, et C. Loisy (dir.). La pédagogie universitaire à l'heure du numérique (p.111-120). De Boeck Université.

Jézégou, A. (2019a) (dir). Traité de la e-Formation. De Boeck Université.

Jézégou, A. (2019b). La distance, la proximité et la présence en e-Formation. Dans A. Jézégou (dir). Traité de la eFormation des adultes (p. 143-163). De Boeck Université.

Jézégou, A. (2019c). L'agentivité humaine en e-Formation des adultes. Dans A. Jézégou (dir). Traité de la e-Formation des adultes (p. 191-211). De Boeck Université.

Jurgenson, N. (2012). When Atoms Meets Bits : Social Media, the Mobil Web and Augmented Revolution. Future Internet, 2012(4), 83-91.

Monteil, J. (1987). À propos du conflit socio-cognitif : d'une heuristique fondamentale à une possible opérationnalisation. Dans R. Beauvois, R. J. Joule et J. Monteil, (dir.), Perspectives cognitives et conduites sociales 1. Théories implicites et conflits cognitifs (p. 199-210). Cousset Delval.

Norman, D. (1999). Affordance, conventions, and design. Interactions, 6(3).

Perret-Clermont, A. N. et Nicolet, M. (2002). Interagir et connaître. Enjeux et régulations sociales dans le développement cognitif. L'Harmattan.

Whiteside, A. L. (2015). Introducing the Social Presence Model to Explore Online and Blended Learning Experiences. Online Learning : Official Journal of the Online Learning Consortium, 19(2). http://olj.onlinelearningconsortium.org/index.php/jaln/article/view/453/137

Whiteside, A. L. (2017). Understanding social presence as a critical literacy. Dans A.L. Whiteside, A.G. Dikkers et K. Swan. (dir). Social Presence in Online Learning: Multiple Perspectives on Practice and Research. Stylus Publishing, 133-142. 